Information Generation as a Functional Basis of Consciousness

Ryota Kanai, Acer Chang, Yen Yu, Ildefons Magrans de Abril, Martin Biehl, \& Nicholas Guttenberg

${ }^{1}$ Araya, Inc., Tokyo, Japan

\title{
Correspondence
}

Ryota Kanai, PhD

Araya, Inc.

Nishi-Shimbashi 2-chome Mori Building 3F,

2 chome-22-1 Nishi-Shimbashi, Minato-ku,

Tokyo, 105-0003

Japan

Tel: +81-3-6435-8885 


\begin{abstract}
What is the biological advantage of having consciousness? Functions of consciousness have been elusive due to the subjective nature of consciousness and ample empirical evidence showing the presence of many nonconscious cognitive performances in the human brain. Drawing upon empirical literature, here, we propose that a core function of consciousness be the ability to internally generate representations of events possibly detached from the current sensory input. Such representations are constructed by generative models learned through sensorymotor interactions with the environment. We argue that the ability to generate information underlies a variety of cognitive functions associated with consciousness such as intention, imagination, planning, short-term memory, attention, curiosity, and creativity, all of which contribute to non-reflexive behaviour. According to this view, consciousness emerged in evolution when organisms gained the ability to perform internal simulations using internal models, which endowed them with flexible intelligent behaviour. To illustrate the notion of information generation, we take variational autoencoders (VAEs) as an analogy and show that information generation corresponds the decoding (or decompression) part of VAEs. In biological brains, we propose that information generation corresponds to top-down predictions in the predictive coding framework. This is compatible with empirical observations that recurrent feedback activations are linked with consciousness whereas feedforward processing alone seems to occur without evoking conscious experience. Taken together, the information generation hypothesis captures many aspects of existing ideas about potential functions of consciousness and provides new perspectives on the functional roles of consciousness.
\end{abstract}




\section{What is the function of consciousness?}

The notion that consciousness is a natural phenomenon suggests that consciousness is subject to a set of universal laws of nature, and a scientific theory of consciousness should correctly identify physical or information theoretic conditions in which consciousness occurs (Chalmers, 1993). This implies that biological brains are not the only medium that gives rise to consciousness. Any physical system that satisfies the necessary and sufficient condition for generating consciousness should possess internal experience (Searle, 2017). However, those conditions have been difficult to determine due to the subjective nature of conscious experience.

Consciousness has both functional and subjective aspects (Block, 1995). The functional aspect, also known as access consciousness, is the objectively observable aspect of consciousness, which are amenable to scientific scrutiny, while the subjective aspect, known as phenomenal consciousness, is not directly observable except for the person experiencing that conscious state. Understanding how phenomenal consciousness occurs within physical systems is known as the Hard problem of consciousness (Chalmers, 1997) and is considered to be one of the toughest problems in science. Much of the interest devoted to possible solutions to the Hard problem often results in the views that consciousness is epiphenomenal, i.e., that subjective experiences exist only as a by-product of information processing without playing any functional role (Velmans, 1991). Instead of directly addressing the Hard problem, a possibly more productive direction might be to consider putative functions of consciousness, namely, cognitive functions that require consciousness in the sense of being awake and able to report stimulus contents with confidence.

In this Opinion article, we examine possible functions of consciousness in the current literature of neuroscience and psychology, and propose that information generation of possibly counterfactual representations is the core faculty of consciousness. Traditionally, many high-level cognitive functions have been associated with consciousness. However, more recent studies have uncovered cognitive functions such as attention (Bahrami, Lavie, \& Rees, 2007; Hsieh, Colas, \& Kanwisher, 2011; Kanai, Tsuchiya, \& Verstraten, 2006; Koch \& Tsuchiya, 2007), working memory (King, Pescetelli, \& Dehaene, 2016; Soto, Mäntylä, \& Silvanto, 2011) and executive control (Lau \& Passingham, 2007) can be performed in the absence of conscious awareness (Lin \& He, 2009). Those empirical findings have made it difficult to pin down cognitive functions that require consciousness. What then is the critical function that requires consciousness? In the literature, there have been a few 
suggestions for cognitive functions that seem to require conscious awareness (Dehaene, Lau, \& Kouider, 2017).

\section{Consciousness is for non-reflexive behaviour}

Reflexive versus non-reflexive behaviour as a marker of consciousness

In clinical settings, the ability to execute non-reflexive behavior is taken as a sign of the presence of consciousness when distinguishing disorders of consciousness: the minimally conscious state (MCS) in which the patient is awake and retains awareness of the self and environment, and the unresponsive wakefulness syndrome (UWS) (Laureys et al., 2010) in which the patient is awake but lacks awareness of the self and environment. According to a modern guideline, MCS is characterized by the presence of cognitively mediated behavior, which is differentiated from reflexive behavior (Giacino et al., 2002). That is, in disorders of consciousness, the presence of consciousness depends on intentional, deliberate behavior as opposed to automated, reflexive behavior triggered solely by the properties of the current sensory input. While identification of non-reflexive behavior is difficult if we were to rely only on behavioral characteristics displayed by a patient, this clinical definition appeals to our intuition that consciousness is needed in nonreflexive behavior.

\section{Bridging a temporal gap in classical conditioning}

Trace conditioning is a kind of classical conditioning which pairs conditioned stimulus (CS) such as a tone with unconditioned stimulus (US), which evoked a response such as an eyeblink from air puffs to the eye. Classical conditioning is said to be a trace conditioning when CS and US do not overlap in time as opposed to a delay conditioning in which CS and US overlap in time. Crucially, empirical evidence suggests that successful trace conditioning requires awareness of the relationship between CS and US, whereas delay conditioning occurs automatically regardless of whether the subject became aware of the relationship (Clark, Manns, \& Squire, 2002; Clark \& Squire, 1998).

The important difference is that in trace conditioning, the subject needs to bridge the temporal gap between CS and US by retaining the information of CS over a blank period. However, the brain cannot prepare automated circuits to associate all possible arbitrary combinations of temporally segregated events, because the number of combinations increases when we consider many different gap durations. Specifically, the information about the CS needs to be maintained over time in the 
brain to be associated with the neural activity evoked by the US. Therefore, the CS needs to be selected as such from many other possible stimuli. That is to say, the subject needs to form a hypothesis about the relationship between CS and US. This contrasts with delay conditioning in which the temporal overlaps allow neural activities evoked by CS and US to directly interact with each other without an additional mechanism to sustain information from the past. As such, the subject does not need to pick a stimulus as a CS to maintain over a short time period. These considerations based on findings in associative learning studies suggests that a possible function of consciousness is to bridge a temporal gap by selecting a small number of events.

\section{Delayed response in a patient with agnosia}

Another clue comes from experiments on the agnosia patient DF who had impairments in conscious object recognition (Goodale, Milner, Jakobson, \& Carey, 1991). When she was asked to indicate the orientation of a slanted slit verbally or by adjusting a handle, she could not report the orientation, suggesting that she had no awareness of the orientation. However, she could post a letter through the slit by adjusting the orientation of the letter in the right angle, suggesting that she could use the orientation information for guiding action. This is a classic example that led to the proposal that the ventral pathway (where DF had a damage) is for conscious vision, whereas the dorsal pathway is for guiding action without necessarily evoking conscious experience.

Crucially, when she was shown the slot first, and then the light was turned off so that she would have to wait for a few seconds before acting, then she failed to reach the slot correctly (Goodale, Jakobson, \& Keillor, 1994; but see Hesse \& Schenk, 2014). This suggests that the unconscious action system needs to be guided online by visual information and to act on offline information retained from the recent past requires the ability to consciously perceive the shape. In other words, 'online systems' that process information real time works without awareness, but to maintain information over time, consciousness is necessary.

These examples suggest that a possible function of consciousness might be to maintain sensory information in short-term memory in a flexible, usable form over a period of time after the stimulus is no longer present. Indeed, based on such observations, Koch proposed a delay test for consciousness in biological systems 
where the presence of consciousness is assessed by the ability to perform a task when a temporal gap is inserted between a stimulus and an action (Koch, 2004).

This is in line with the second and the third laws of the three laws of qualia - a framework proposed by Ramachandran (Ramachandran \& Hirstein, 1997). 'Qualia' refers to the qualitative aspect of conscious experience, which is sometimes characterized as the redness of a sunset or the painfulness of headache (Kanai \& Tsuchiya, 2012). In the three laws of qualia, qualia are characterized by three functional properties, namely, 1) irrevocability, 2) flexibility and 3) short-term memory. Irrevocability refers to the observation that we cannot override the contents of experience at will via top-down attention. Flexibility implies the fact that once sensory events are consciously registered, the contents can be used for multiple, open-ended purposes unlike reflexive behavior. Short-term memory refers to the fact that the content of consciousness remains for a sufficient time to allow interaction with the executive system for making choices.

\section{Counterfactual predictions for intention and planning}

Another important aspect of consciousness pertains to motor behavior such as intention or planning. Intention can be formulated as predictions about the consequences of actions before those actions are executed (Jeannerod, 1994). For example, I can predict my sensory inputs from visual and somatosensory systems that would arise if I were to make a particular action such as waving my hand in front of my face even without actually performing the movement. I can also think about going to the kitchen to make coffee without actually standing up from the couch in the living room. These instances of intention or planning are counterfactual predictions in the sense that planned actions have not materialized in the reality while imagining a possible future.

The importance of consciousness in planning has been argued in the literature. More than 20 years ago, Crick and Koch discussed plausible functions of visual awareness are to produce the best interpretation of the current scene, and to make the information available for voluntary actions after a delay (Crick \& Koch, 1998; Crick \& Koch, 1995). Based on this view, they once proposed that neurons in the primary visual cortex (V1) do not directly produce conscious experience (Crick \& Koch, 1995). The rationale for the $V 1$ hypothesis comes from the consideration that the biological utility of conscious perception is to make the sensory information available for planning and executing voluntary motor outputs. When we consciously 
experience sensory information (e.g. seeing a red ball), we can use that information for multiple purposes such as planning future actions. Crucially, the V1 hypothesis predicted that neuronal activities in V1 do not directly contribute to conscious experience because $\mathrm{V} 1$ neurons do not directly project to the prefrontal cortex implicated in the function of planning. While the position of one of the original proposers may have changed (Koch, Massimini, Boly, \& Tononi, 2016), we believe that the rationale that a functional consequence of conscious perception is the availability of the information for future planning is still valid independent of his current position.

The idea is also compatible with the current global workspace theory in which the role of consciousness is to make sensory information shared across multiple cortical regions including the fronto-parietal networks (Baars, 2005; Baars, 1997; Dehaene, Kerszberg, \& Changeux, 1998; Dehaene \& Naccache, 2001). The key idea is that consciousness allows one to utilize the content of conscious perception for flexible purposes (Earl, 2014). These hypotheses put emphasis on short-term memory, working memory, planning and executive control as functional capabilities endowed by consciousness. In other words, a function of consciousness is to make information globally available across the system.

Another possible role of consciousness is metacognition. A wide range of cognitive functions are known to be performed without awareness. As psychophysical and neurological studies have shown, objective performance in a visual task can be above chance while the subject claims to have no awareness of seeing a stimulus. Thus, it is thought that a higher-level, meta-representation of a first-order representation of a sensory stimulus should be needed for consciousness. This idea is closely related to the notion of higher-order theories of consciousness (Carruthers, 2005; Lau \& Rosenthal, 2011; Rosenthal, 2006). In line with higher-order theories, confidence ratings are often used as a proxy measure for the presence of conscious perception (Fleming \& Lau, 2014; Sherman, Barrett, \& Kanai, 2015).

These two aspects of consciousness, i.e., broadcasting of information and metacognition, have been summarised in a recent paper (Dehaene et al., 2017) as C1 and C2, respectively. The functions of consciousness discussed in this Opinion article mainly concerns $\mathrm{C} 1$, and our information generation hypothesis proposes that what underlies a variety of cognitive functions such as short-term memory, intention, 
and planning is the capability of generating fictional representations using internal, sensorimotor models.

Taken together, we propose that a key function of consciousness is to allow nonreflexive behavior such as responding after a delay, or executing an action based on internally generated plans. Is there a computational mechanism that is common across a variety of non-reflexive behavior?

\section{Counterfactual Information generation theory of consciousness}

Here, we suggest that a common thread across those examples of non-reflexive behavior is information generation. It's the ability to internally generate sensory representations that are not direct reflections of the current sensory input. They could be counterfactual representations since information maintained over time in shortterm memory from the past or predictions of sensory consequences of unexecuted future actions do not necessarily correspond to events actually happening in the present environment.

Our hypothesis is that this ability to generate possibly counterfactual representations using internal models learned through interactions with the environment is the function of consciousness. This hypothesis, which we call information generation hypothesis, helps us understand functional advantages of consciousness and predict under what kind of tasks consciousness would be required.

The ability to generate internal representations independent of current sensory inputs allows an agent to engage with possibly counterfactual states not happening at the present moment. This enables an agent to detach itself from the environment and perform non-reflexive behavior such as planning future actions through mental simulations of the environment (Grush, 2004). Furthermore, it allows an agent even to learn from fictional scenarios it has never experienced (Ha \& Schmidhuber, 2018). This capability provides an enormous advantage to organisms for survival.

\section{Counterfactuals in model-based reinforcement learning}

The functional advantage of internal simulation is also apparent in reinforcement learning. In the literature of reinforcement learning, learning strategy is divided into model-free and model-based (Dayan \& Berridge, 2014). Model-free reinforcement learning such as Q-learning learns to derive the best action under the current state but does not involve direct learning of the structure of the environment. Model-based 
reinforcement learning captures the sequential contingencies of events and actions, namely, the predictive model of future states for a given action. This model-based approach allows an agent to use the knowledge of the structure of the environment to plan a course of action. For example, a bird's eye model of a maze would allow an agent to plan for the optimal path through an internal simulation. The model-based approach thus affords an agent with the ability to compute an optimal sequence of actions through mental simulation using their internal models. In both biological and artificial intelligence, generative models of action-state sequences play an essential role in model-based reinforcement learning. For example, Dyna proposed by Sutton (Sutton, 1991) adopts the idea that planning is 'trying things in your head'. Crucially, the model-based approach allows an agent to adapt to new goals flexibly because it can use the internal model to optimize its behavior without trial and error.

\section{Intrinsic motivation, saliency and infotaxis}

Predictions of future states are useful for sampling information from the environment efficiently. This is because such generated future states allow an agent to estimate various statistical quantities of interest in a given context. For example, an agent can compute in a model-based manner what would be the expected information gain (Schmidhuber, 1991, 2010) or other forms of intrinsic motivation (Oudeyer, 2007) in response to future (and therefore counterfactual) actions. The framework of active inference (Friston et al., 2015; Friston, Samothrakis, \& Montague, 2012; Friston et al., 2013) promises to treat future actions in a similar way as other counterfactual events like hidden causes and infer them by conditioning on an optimized utility function. While this utility function usually corresponds to expected free energy it has been shown that the same approach can be equally applied to various other intrinsic motivations (Biehl, Guckelsberger, Salge, Smith, \& Polani, 2018). This suggests that the information generation perspective may be able to naturally incorporate current research on intrinsic motivations as well.

In the context of attention and eye movement, expected information gain can be considered as a saliency map (Friston, Adams, Perrinet, \& Breakspear, 2012). Such formulation is considered model-based in the sense that the saliency (i.e. expected decrease in the entropy of hidden states) is computed based on a generative model of the sensory input given the underlying hidden cause. Infotaxis is another such example where search behavior is guided by expected information gain given an action and is likely to be found in many creatures (Vergassola, Villermaux, \& Shraiman, 2007). These model-based search strategies are important when signals 
are sparse, and highlight the utility of counterfactual predictions in biological systems (Friston et al., 2015).

Taken together, our analysis suggests that the ability to generate information enables an agent to perform mental simulations for planning future action sequences, which would be otherwise difficult only with a collection of reflexive behaviors. This ability underlies model-based reinforcement learning and intrinsic motivations such as curiosity and infotaxis.

\section{What does it mean to internally generate information?}

So far, we have argued that the ability to generate counterfactual representations is the core function of consciousness, allowing an agent to perform non-reflexive behaviors. However, we have not specified what kind of neural processing should be considered as generating information.

Here we argue that information generation can be seen as production of sensory representations using internal models. This is achieved by reversing the process of representation learning, which is projection from sensory inputs to internal models. In what follows, we will illustrate the concept of information generation using modern approaches to constructing generative models with deep neural networks.

\section{Generative models in deep neural networks}

Many sophisticated methods such as Generative Adversarial Networks (GANs) (Goodfellow et al., 2014; Gulrajani, Ahmed, Arjovsky, Dumoulin, \& Courville, 2017; Hindupur, 2017) or Variational Autoencoders (VAEs) (Kingma \& Welling, 2013) for training models for generating sensory representations have been developed in recent years, but they have not received sufficient attention in the context of neuroscience. While the concept of learning a generative model - statistical model of the process of how sensory data are generated from a set of hidden causes - is common in interpreting computational principles of the brain, what it means for the brain to use generative models for producing representations has rarely been considered. Here, we illustrate the idea of information generation using the (variational) autoencoder as a metaphor and relate it to the predictive coding architecture in the brain.

VAEs are a class of neural networks consisting of an encoder/inference network and a decoder/generative network (Figure 1a). The encoder works as data compression 
by transforming the input data (e.g. image) to abstract, latent representations of low dimensionality (i.e. parameters of a Gaussian distribution). The decoder then converts the abstract representation back to the original data space as the output. The networks are trained so that the output matches the input as closely as possible, namely, maximizing the lower bound of the probability of generating real data samples. Once trained successfully, the decoder network can be used independently of the encoder network, and thus without the input, to generate representations in the data space (e.g. see Figure 1). This property of the decoder network enables production of novel and counterfactual representations based on previous experiences. The loss function of VAE is known as the evidence lower bound, and is related to in Friston's free energy principle (Friston, 2009, 2010).

Under the computational scheme of VAEs, the decoder/generative and encoder/inference networks correspond to the generation and processing of information, respectively (Figure 1a). The decoder/generative network can be used for generating representations in the data space by choosing a state in the latent space, which allows production of counterfactual representations including those the agent has never encountered before. From the architecture of VAEs, we can formulate information generation in terms of mapping from an abstract low dimensional representation to a high dimensional representation in the data (i.e. sensory) space.

\section{Predictive coding framework of the brain}

However, the architecture of the VAEs does not resemble the networks found in the biological brain. How do those encoder and decoder networks in VAEs correspond to the network architectures in biological brains? To establish correspondences with the brain, we consider hierarchical predictive coding (Friston \& Kiebel, 2009; Hohwy, 2014; Rao \& Ballard, 1999). According to this view, representations at a higher layer serves as a prediction for a lower layer, and this is communicated via top-down signals, whereas prediction errors (i.e., the discrepancy between the top-down prediction and bottom-up signals) are communicated back to the higher layer to update the prediction.

At a conceptual level, the reciprocal interactions between top-down prediction and bottom-up prediction error in the predictive coding framework can be roughly mapped to the decoder and encoder in VAEs, respectively (figure 1b). While whether the 
brain utilizes the predictive coding architecture is still a matter of debate, and there are multiple ways to implement similar architectures (e.g. Heeger, 2017), the framework provides a link between the encoder/decoder scheme presented above and possible counterparts in the biological brain. That is, information generation, which we claim to be crucial for consciousness, is mediated by top-down prediction, whereas the bottom-up message passing of prediction errors corresponds to encoding (or data compression). By interpreting feedback predictions as information generation, our current hypothesis predicts that consciousness is generated by the feedback predictions. This is in line with multiple observations that reports of conscious perception is associated with additional feedback activations from higher sensory areas to lower sensory areas (Lamme, Supèr, Landman, Roelfsema, \& Spekreijse, 2000; Manita et al., 2015; Supèr, Spekreijse, \& Lamme, 2001).

Note, however, that information generation could in principle occur both in feedforward and feedback networks, as the example of VAE consists of purely feedforward networks. Recurrent connections are just a specific case of implementing information generation in the brain and are not a pre-requisite for information generation. Therefore, the information generation hypothesis predicts that consciousness could be also produced in simple networks like the VAE as long as they generate information (see next section for a related issue). While we acknowledge that some readers might find it unlikely that such a simple network possesses consciousness, we leave this possibility open for future discussion.

\section{Information Generation from an Intrinsic Perspective}

We have attributed information generation to the decoder/generative network in VAEs and top-down feedback in predictive coding networks. However, these attributions are made from an extrinsic viewpoint. If we are given an arbitrary network of neurons, it would be difficult to determine which part of the network corresponds to a decoder or an encoder. Is there a principled way to determine whether a system is generating information? One noticeable characteristic that distinguishes the decoder network from the encoder network is that in the decoder, the network has a divergent structure from a low dimensional representation to a higher dimensional representation, whereas in the encoder network, the network has a convergent structure. Further work is needed to determine which part of a network should be considered as generating information from an intrinsic perspective without requiring an extrinsic interpreter. 


\section{Related Theories}

In this Opinion paper, we argued that a potential function of consciousness is to generate potentially counterfactual representations, and this function allows flexible response mechanisms for various forms of non-reflexive, goal directed behaviour. As discussed earlier, our idea is compatible with putative functions of consciousness proposed by various authors in the context of the global workspace theory (Baars, 2005b; Dehaene et al., 1998), V1 hypothesis (Crick \& Koch, 1995), and the three laws of qualia (Ramachandran \& Hirstein, 1997), in which flexible use of sensory information is thought to be a key element of consciousness. Also a very similar idea has been also proposed by Pennartz that consciousness is for deliberate decision making on goal-directed behaviour requiring internal generation of representations (Pennartz, 2018). In what follows, we briefly overview other theories that are relevant for our current hypothesis.

\section{Semantic Pointer Competition}

A notion similar to the decoder networks in our hypothesis has been proposed in the context of the Semantic Pointer Competition (SPC) hypothesis (Thagard \& Stewart, 2014). SPC proposes that consciousness is supported by representations, semantic pointers and competition among semantic pointers. In brief, SPC posits that neural representations constructed for each modality (e.g. sensory, motor, and emotional) are combined to form the so-called semantic pointer, which works as a symbol that bundles associated representations coming from multiple modalities. The content of consciousness is determined through competitive mechanisms among semantic pointers towards a limited capacity. In this hypothesis, semantic pointers play two functions. One is to make predictions/inferences about other semantic pointers and the other is to unpack specific contents in the representation coming from a specific modality. The information generation process in our proposal offers a more concrete implementation of this unpacking process. A key theoretical difference is that the information generation hypothesis claims that this unpacking process corresponds to the generation of conscious experience, whereas SPC takes the whole architecture consisting of the three components (i.e. representations, semantic pointers and competition) to be the foundation of consciousness.

\section{Regret}

Frith and Metzinger proposed that a function of consciousness is associated with the notion of regret (Frith \& Metzinger, 2016). Regret implies extension of the self across time because regret is about the action taken in the past or anticipation of regret in 
the future. This hypothesis built around the notion of regret also includes other functions of consciousness such as the ability to communicate subjective experience with others, the ability to distinguish the real present from imaginary possible desirable states as used in future planning, and persistence of the self across time through the model of the self. Information generation discussed in our paper should be considered as a way to implement mechanisms to compute regret through production of and interactions with counterfactual past and future. The ability to evaluate counterfactual regret would serve the purpose of learning causal relationship between action and consequences (Pearl \& Mackenzie, 2018).

\section{Qualia: Factual versus Counterfactual Representations}

Several authors have argued that one important function of conscious experience is the ability to distinguish between representations of factual reality of the here and now and counterfactual possibilities (Frith \& Metzinger, 2016; Gregory, 1998; Kanai \& Tsuchiya, 2012). While we have emphasised the link between potentially counterfactual representations and consciousness, conscious experience is more vivid for the actual sensory input. How can the information generation hypothesis explain the stronger conscious experience for factual events than those for counterfactual possibilities?

Our interpretation is that conscious experience of the current sensory input is also a product of the internal, generative models. But instead of generating counterfactual events, the same generative networks produce a representation of the current input. The differences in vividness of experience come from the differences in the degree of details produced by the generative models. When counterfactual representations are generated (e.g. imagining a face), generative networks can specify only up to a certain level of details, whereas when bottom-up signals are available (e.g. seeing a face), generative networks can utilize those signals to produce representations with finer details. We speculate that this difference in the level of details in internally generated images correspond to the differences in the vividness between actual and counterfactual experiences and serves the purpose of distinguishing factual and counterfactual representations.

Furthermore, there has been a suggestion that counterfactual representations implicit in the internal models of sensorimotor contingencies contribute to the 'presence' of an object of perception (Seth, 2014). According to this theory, the perceived presence associated with a stimulus is supported by counterfactually-rich generative 
models that have learned sensorimotor contingencies. Given the importance of perceptual presence in conscious experience of here and now, numerous possibilities stored in the structure of the generative models may shape the ineffable richness of qualia.

\section{Origins of Conscious Mind}

While consciousness and intelligence are generally distinguished as separate entities, our hypothesis suggests a possible link between the intelligent behaviour endowed by flexible use of internal sensorimotor models and consciousness.

What is the functional benefit of having the ability to generate counterfactual representations? Dennett's formulation of stages of evolution of minds (Dennett, 1996) illustrates the benefit of counterfactuals in evolution and intelligence. Dennett proposed useful distinctions among different stages of the evolution of creatures. These include the following four types of creatures.

The first stage of creatures is called Darwinian. Darwinian creatures are the most primitive form of creatures. The organisms at this stage do not learn from experiences but have a fixed set of behaviours determined by their genetic composition. They adapt to the environment only as a species through natural selection and mutation to their genes. Thus, learning occurs at a population level but not as individual agents.

The second stage is called Skinnerian. Skinnerian creatures - named after the psychologist Skinner - learn from their own experience through associative learning. If a particular action led to a reward (e.g. food), then that action is reinforced, whereas an action that led to an aversive result will be avoided. Skinnerian creatures can adapt to the environment during the life time of individual organisms through trial and error. However, there is a limitation in that they can only learn from their own experiences. They would have to learn danger from surviving dangerous situations.

Then, there comes the third stage of creatures - Popperian creatures named after the philosopher Popper. They have internal simulation of the environment and select an action based on predictions about simulated consequences of repertoires of future action sequences. This ability allows Popperian creatures to learn from their simulated, counterfactual experience, and to select reasonable actions even in a new environment when their internal model approximates it reasonably well. Moreover, 
the ability to form counterfactual representations of sensory consequences contingent upon their future action is the core function of intention. The information generation theory of consciousness suggests that consciousness appears at this stage of the evolution of the mind. In other words, our theory suggests the origin of consciousness is the functional advantage of having counterfactual predictions using an internal model of the environment, as it allows Popperian creatures to simulate consequences of possible actions and avoid executing actions that could be dangerous for survival.

Finally, the fourth stage is called Gregorian. Gregorian creatures can learn from their cultural environment through words and language. This requires the ability to run a simulation of the environment using information they learned through books and the Internet. In Popperian creatures, internal models had to be built upon their own experiences. Gregorian creatures on the other hand can improve their internal models through communication with other agents, which endows them with an additional leverage of intelligence. Here, the ability to internally represent counterfactual states is a prerequisite.

Our current hypothesis predicts that the presence of consciousness should be measured by the presence of internal generative models of the environment and the self. Moreover, the hypothesis predicts that model-based action selection should require consciousness. Is model-based reinforcement learning or other forms of model-based decision making possible without consciousness? One important future direction suggested by these predictions is to establish a formal computational and experimental framework to determine whether a given system relies on information generated by its internal generative models. In the example of VAE, we argued that dimensional expansion might be the essence of information generation. However, we need to define information generation more formally as an intrinsic property of a system. Further work is needed to establish such formalisms.

\section{Recapitulation}

To recapitulate, we set off to identify functions of consciousness by examining cognitive tasks that appear to require consciousness. Based on a few examples such as trace conditioning and delayed, non-reflexive responses, we aimed to extract functional essences underlying those tasks, and hypothesised that a function of consciousness is to bridge the temporal gap by internally generating representations of stimuli that are no longer present in the proximal environment. While the 
hypothesis emphasised the counterfactual aspect of generated information, it considers the conscious perception of current stimuli also as a product of internal generation. To illustrate the processes underlying information generation, we discussed information generation in the decoder networks in VAEs and speculated that in the brain, feedback connections should correspond to such processes. Finally, we reversed the logic of necessity and sufficiency and postulated a stronger version of our hypothesis that a system with the ability to generate representations detached from the current sensory input must have consciousness.

Note that there are two levels hypotheses within our information generation hypothesis. A weak version of the hypothesis simply claims that a function of consciousness is to generate and maintain representations of events detached from the current sensory input. This version predicts that tasks in which correct responses are not immediately available thereby requiring interactions with internally generated representations depend on consciousness. In other words, the weaker version claims that information generation is necessary for a system to perform consciousness dependent tasks. As such, the ability to perform a task that require internal generation of information is considered as an indicator of consciousness.

On the other hand, a stronger version of the hypothesis goes further and predicts that machines with similar functionalities would also possess consciousness even if they are relatively simple to build. This implies that that all tasks that require counterfactual information generation also require consciousness. Therefore, consciousness and counterfactual information generation cannot be distinguished and we consider them identical. In other words, the stronger version claims that counterfactual information generation is sufficient for a system to be conscious. While readers may find this possibility unlikely, we argue that this possibility should be left open for further studies and the conceptual distinction of the two versions of the hypothesis would be useful for further examining this hypothesis in light of future research.

\section{Concluding Remarks}

In summary, we proposed that the ability to generate possibly counterfactual information underlies a variety of cognitive functions enabled by consciousness such as intention, imagination, planning, short-term memory, attention, curiosity, and creativity, all of which contribute to non-reflexive, behavioural flexibility. We further hypothesized that information generation corresponds to feedback predictions in the 
brain using the predictive coding framework as a model of the brain. Further studies are needed to formally define information generation as an intrinsic property of biological and artificial systems.

\section{Acknowledgements}

The author thanks the members of Araya for discussion. R.K., Y.Y. and IMA are funded by Japan Science and Technology Agency (JST) CREST project. R.K. and M.B. are supported by Templeton World Charity Foundation. 


\section{References}

Baars, Bernard J. (2005a). Global workspace theory of consciousness: Toward a cognitive neuroscience of human experience. Progress in Brain Research, 150, 45-53. https://doi.org/10.1016/S0079-6123(05)50004-9

Baars, Bernard J. (2005b). Global workspace theory of consciousness: Toward a cognitive neuroscience of human experience. Progress in Brain Research, 150, 45-53. https://doi.org/10.1016/S0079-6123(05)50004-9

Baars, B.J. (1997). In the theatre of consciousness. Global Workspace Theory, a rigorous scientific theory of consciousness. Journal of Consciousness Studies, 4(4), 292-309.

Bahrami, B., Lavie, N., \& Rees, G. (2007). Attentional Load Modulates Responses of Human Primary Visual Cortex to Invisible Stimuli. Current Biology, 17(6), 509-513. https://doi.org/10.1016/j.cub.2007.01.070

Biehl, M., Guckelsberger, C., Salge, C., Smith, S. C., \& Polani, D. (2018). Expanding the Active Inference Landscape: More Intrinsic Motivations in the PerceptionAction Loop. Frontiers in Neurorobotics, Vol.12. https://doi.org/10.3389/fnbot.2018.00045 Block, N. (1995). On a confusion about a function of consciousness. Behavioral and Brain Sciences, 18(2), 227-247. https://doi.org/10.1017/S0140525X00038188

Carruthers, P. (2005). Consciousness: Essays from a Higher-Order Perspective. Oxford, New York: Oxford University Press.

Chalmers, D. J. (1993). Toward a Theory of Consciousness. Indiana University.

Chalmers, D. J. (1997). The conscious mind: In search of a fundamental theory (1. issued as an Oxford University Press paperback). New York: Oxford Univ. Press.

Clark, R. E., Manns, J. R., \& Squire, L. R. (2002). Classical conditioning, awareness, and brain systems. Trends in Cognitive Sciences, 6(12), 524-531. https://doi.org/10.1016/S1364-6613(02)02041-7

Clark, R. E., \& Squire, L. R. (1998). Classical Conditioning and Brain Systems: The Role of Awareness. Science, 280(5360), 77-81. https://doi.org/10.1126/science.280.5360.77

Crick, F., \& Koch, C. (1998). Consciousness and neuroscience. Cerebral Cortex, 8(2), 97-107. https://doi.org/10.1093/cercor/8.2.97

Crick, Francis, \& Koch, C. (1995). Are we aware of neural activity in primary visual cortex? Nature, 375(6527), 121-123. https://doi.org/10.1038/375121a0

Dayan, P., \& Berridge, K. C. (2014). Model-based and model-free Pavlovian reward learning: Revaluation, revision, and revelation. Cognitive, Affective, \& Behavioral Neuroscience, 14(2), 473-492. https://doi.org/10.3758/s13415014-0277-8

Dehaene, S., Kerszberg, M., \& Changeux, J.-P. (1998). A neuronal model of a global workspace in effortful cognitive tasks. Proceedings of the National Academy of Sciences, 95(24), 14529-14534. https://doi.org/10.1073/pnas.95.24.14529

Dehaene, S., Lau, H., \& Kouider, S. (2017). What is consciousness, and could machines have it? Science, 358(6362), 486-492. https://doi.org/10.1126/science.aan8871

Dehaene, S., \& Naccache, L. (2001). Towards a cognitive neuroscience of consciousness: Basic evidence and a workspace framework. Cognition, 79(1), 1-37. https://doi.org/10.1016/S0010-0277(00)00123-2

Dennett, D. C. (1996). Kinds of minds: Toward an understanding of consciousness. New York: BasicBooks. 
Earl, B. (2014). The biological function of consciousness. Frontiers in Psychology, 5. https://doi.org/10.3389/fpsyg.2014.00697

Fleming, S. M., \& Lau, H. C. (2014). How to measure metacognition. Frontiers in Human Neuroscience, 8. https://doi.org/10.3389/fnhum.2014.00443

Friston, K. (2009). The free-energy principle: A rough guide to the brain? Trends in Cognitive Sciences, 13(7), 293-301. https://doi.org/10.1016/j.tics.2009.04.005

Friston, K. (2010). The free-energy principle: A unified brain theory? Nature Reviews Neuroscience, 11(2), 127-138. https://doi.org/10.1038/nrn2787

Friston, K., Adams, R., Perrinet, L., \& Breakspear, M. (2012). Perceptions as Hypotheses: Saccades as Experiments. Frontiers in Psychology, 3. https://doi.org/10.3389/fpsyg.2012.00151

Friston, K., \& Kiebel, S. (2009). Predictive coding under the free-energy principle. Philosophical Transactions of the Royal Society B: Biological Sciences, 364(1521), 1211-1221. https://doi.org/10.1098/rstb.2008.0300

Friston, K., Rigoli, F., Ognibene, D., Mathys, C., Fitzgerald, T., \& Pezzulo, G. (2015). Active inference and epistemic value. Cognitive Neuroscience, 6(4), 187-214. https://doi.org/10.1080/17588928.2015.1020053

Friston, K., Samothrakis, S., \& Montague, R. (2012). Active inference and agency: Optimal control without cost functions. Biological Cybernetics, 106(8), 523541. https://doi.org/10.1007/s00422-012-0512-8

Friston, K., Schwartenbeck, P., Fitzgerald, T., Moutoussis, M., Behrens, T., \& Dolan, R. J. (2013). The anatomy of choice: Active inference and agency. Frontiers in Human Neuroscience, 7. https://doi.org/10.3389/fnhum.2013.00598

Frith, C. D., \& Metzinger, T. (2016). What's the Use of Consciousness?: How the Stab of Conscience Made Us Really Conscious. Retrieved from https://mitpress.universitypressscholarship.com/view/10.7551/mitpress/97802 62034326.001.0001/upso-9780262034326-chapter-12

Giacino, J. T., Ashwal, S., Childs, N., Cranford, R., Jennett, B., Katz, D. I., ... Zasler, N. D. (2002). The minimally conscious state: Definition and diagnostic criteria. Neurology, 58(3), 349-353.

Goodale, M. A., Jakobson, L. S., \& Keillor, J. M. (1994). Differences in the visual control of pantomimed and natural grasping movements. Neuropsychologia, 32(10), 1159-1178.

Goodale, M. A., Milner, A. D., Jakobson, L. S., \& Carey, D. P. (1991). A neurological dissociation between perceiving objects and grasping them. Nature, 349(6305), 154-156. https://doi.org/10.1038/349154a0

Goodfellow, I., Pouget-Abadie, J., Mirza, M., Xu, B., Warde-Farley, D., Ozair, S., ... Bengio, Y. (2014). Generative Adversarial Nets. In Z. Ghahramani, M. Welling, C. Cortes, N. D. Lawrence, \& K. Q. Weinberger (Eds.), Advances in Neural Information Processing Systems 27 (pp. 2672-2680). Retrieved from http://papers.nips.cc/paper/5423-generative-adversarial-nets.pdf

Gregory, R. (1998). Brainy mind. BMJ (Clinical Research Ed.), 317(7174), 16931695. https://doi.org/10.1136/bmj.317.7174.1693

Grush, R. (2004). The Emulation Theory of Representation: Motor Control, Imagery, and Perception. Behavioral and Brain Sciences, 27(3), 377-396.

Gulrajani, I., Ahmed, F., Arjovsky, M., Dumoulin, V., \& Courville, A. (2017). Improved Training of Wasserstein GANs. ArXiv:1704.00028 [Cs, Stat]. Retrieved from http://arxiv.org/abs/1704.00028

Ha, D., \& Schmidhuber, J. (2018). World Models. ArXiv:1803.10122 [Cs, Stat]. https://doi.org/10.5281/zenodo.1207631

Heeger, D. J. (2017). Theory of cortical function. Proceedings of the National Academy of Sciences, 114(8), 1773-1782. https://doi.org/10.1073/pnas.1619788114 
Hesse, C., \& Schenk, T. (2014). Delayed action does not always require the ventral stream: A study on a patient with visual form agnosia. Cortex, 54, 77-91. https://doi.org/10.1016/j.cortex.2014.02.011

Hindupur, A. (2017, April 19). The GAN Zoo. Retrieved from Deep Hunt website: https://deephunt.in/the-gan-zoo-79597dc8c347

Hohwy, J. (2014). The Predictive Mind (1 edition). Oxford, United Kingdom ; New York, NY, United States of America: Oxford University Press.

Hsieh, P.-J., Colas, J. T., \& Kanwisher, N. (2011). Pop-Out Without Awareness: Unseen Feature Singletons Capture Attention Only When Top-Down Attention Is Available. Psychological Science, 22(9), 1220-1226. https://doi.org/10.1177/0956797611419302

Jeannerod, M. (1994). The representing brain: Neural correlates of motor intention and imagery. Behavioral and Brain Sciences, 17(2), 187-202. https://doi.org/10.1017/S0140525X00034026

Kanai, R., \& Tsuchiya, N. (2012). Qualia. Current Biology, 22(10), R392-R396. https://doi.org/10.1016/j.cub.2012.03.033

Kanai, R., Tsuchiya, N., \& Verstraten, F. A. J. (2006). The scope and limits of topdown attention in unconscious visual processing. Current Biology: $C B$, 16(23), 2332-2336. https://doi.org/10.1016/j.cub.2006.10.001

King, J.-R., Pescetelli, N., \& Dehaene, S. (2016). Brain Mechanisms Underlying the Brief Maintenance of Seen and Unseen Sensory Information. Neuron, 92(5), 1122-1134. https://doi.org/10.1016/j.neuron.2016.10.051

Kingma, D. P., \& Welling, M. (2013). Auto-Encoding Variational Bayes. ArXiv:1312.6114 [Cs, Stat]. Retrieved from http://arxiv.org/abs/1312.6114

Koch, C. (2004). The Quest for Consciousness: A Neurobiological Approach (1 edition). Denver, Colo: Roberts \& Co.

Koch, C., Massimini, M., Boly, M., \& Tononi, G. (2016). Neural correlates of consciousness: Progress and problems. Nature Reviews Neuroscience, 17(5), 307-321. https://doi.org/10.1038/nrn.2016.22

Koch, C., \& Tsuchiya, N. (2007). Attention and consciousness: Two distinct brain processes. Trends in Cognitive Sciences, 11(1), 16-22. https://doi.org/10.1016/j.tics.2006.10.012

Lamme, V. A. F., Supèr, H., Landman, R., Roelfsema, P. R., \& Spekreijse, H. (2000). The role of primary visual cortex (V1) in visual awareness. Vision Research, 40(10), 1507-1521. https://doi.org/10.1016/S0042-6989(99)00243-6

Lau, H. C., \& Passingham, R. E. (2007). Unconscious Activation of the Cognitive Control System in the Human Prefrontal Cortex. Journal of Neuroscience, 27(21), 5805-5811. https://doi.org/10.1523/JNEUROSCI.4335-06.2007

Lau, H., \& Rosenthal, D. (2011). Empirical support for higher-order theories of conscious awareness. Trends in Cognitive Sciences, 15(8), 365-373. https://doi.org/10.1016/j.tics.2011.05.009

Laureys, S., Celesia, G. G., Cohadon, F., Lavrijsen, J., León-Carrión, J., Sannita, W. G., ... the European Task Force on Disorders of Consciousness. (2010). Unresponsive wakefulness syndrome: A new name for the vegetative state or apallic syndrome. BMC Medicine, 8(1), 68. https://doi.org/10.1186/17417015-8-68

Lin, Z., \& He, S. (2009). Seeing the invisible: The scope and limits of unconscious processing in binocular rivalry. Progress in Neurobiology, 87(4), 195-211. https://doi.org/10.1016/j.pneurobio.2008.09.002

Manita, S., Suzuki, T., Homma, C., Matsumoto, T., Odagawa, M., Yamada, K., ... Murayama, M. (2015). A Top-Down Cortical Circuit for Accurate Sensory Perception. Neuron, 86(5), 1304-1316. https://doi.org/10.1016/j.neuron.2015.05.006

Maximally informative foraging by Caenorhabditis elegans | eLife Lens. (n.d.). Retrieved 22 July 2017, from https://lens.elifesciences.org/04220/ 
Oudeyer, P.-Y. (2007). What is intrinsic motivation? A typology of computational approaches. Frontiers in Neurorobotics, 1. https://doi.org/10.3389/neuro.12.006.2007

Pearl, J., \& Mackenzie, D. (2018). The Book of Why: The New Science of Cause and Effect (1 edition). New York: Basic Books.

Pennartz, C. M. A. (2018). Consciousness, Representation, Action: The Importance of Being Goal-Directed. Trends in Cognitive Sciences, 22(2), 137-153. https://doi.org/10.1016/j.tics.2017.10.006

Ramachandran, V. S., \& Hirstein, W. (1997). Three laws of qualia: What neurology tells us about the biological functions of consciousness. Journal of Consciousness Studies, 4(5-6), 429-457.

Rao, R. P., \& Ballard, D. H. (1999). Predictive coding in the visual cortex: A functional interpretation of some extra-classical receptive-field effects. Nature Neuroscience, 2(1), 79-87. https://doi.org/10.1038/4580

Rosenthal, D. (2006). Consciousness and Mind (1 edition). Oxford ; New York: Clarendon Press.

Schmidhuber, J. (1991). Curious model-building control systems. [Proceedings] 1991 IEEE International Joint Conference on Neural Networks, 1458-1463 vol.2. https://doi.org/10.1109/IJCNN.1991.170605

Schmidhuber, J. (2010). Formal Theory of Creativity, Fun, and Intrinsic Motivation (1990 \#x2013;2010). IEEE Transactions on Autonomous Mental Development, 2(3), 230-247. https://doi.org/10.1109/TAMD.2010.2056368

Searle, J. (2017). Biological Naturalism. In S. Schneider \& M. Velmans (Eds.), The Blackwell Companion to Consciousness (pp. 327-336). https://doi.org/10.1002/9781119132363.ch23

Seth, A. (2014). A predictive processing theory of sensorimotor contingencies: Explaining the puzzle of perceptual presence and its absence in synesthesia. Cognitive Neuroscience, 5 (2), 97-118.

Sherman, M., Barrett, A. B., \& Kanai, R. (2015). Inferences about consciousness using subjective reports of confidence. In M. Overgaard (Ed.), Behavioral Methods in Consciousness Research (pp. 87-106). https://doi.org/10.1093/acprof:oso/9780199688890.003.0006

Soto, D., Mäntylä, T., \& Silvanto, J. (2011). Working memory without consciousness. Current Biology, 21(22), R912-R913. https://doi.org/10.1016/j.cub.2011.09.049

Supèr, H., Spekreijse, H., \& Lamme, V. A. (2001). Two distinct modes of sensory processing observed in monkey primary visual cortex (V1). Nature Neuroscience, 4(3), 304-310. https://doi.org/10.1038/85170

Sutton, R. S. (1991). Dyna, an integrated architecture for learning, planning, and reacting. ACM SIGART Bulletin, 2(4), 160-163. https://doi.org/10.1145/122344.122377

Thagard, P., \& Stewart, T. C. (2014). Two theories of consciousness: Semantic pointer competition vs. information integration. Consciousness and Cognition, 30, 73-90. https://doi.org/10.1016/j.concog.2014.07.001

Velmans, M. (1991). Is human information processing conscious? Behavioral and Brain Sciences, 14(4), 651-669. https://doi.org/10.1017/S0140525X00071776

Vergassola, M., Villermaux, E., \& Shraiman, B. I. (2007). 'Infotaxis' as a strategy for searching without gradients. Nature, 445(7126), 406-409. https://doi.org/10.1038/nature05464 


\section{Figure 1}

a) Autoencoder

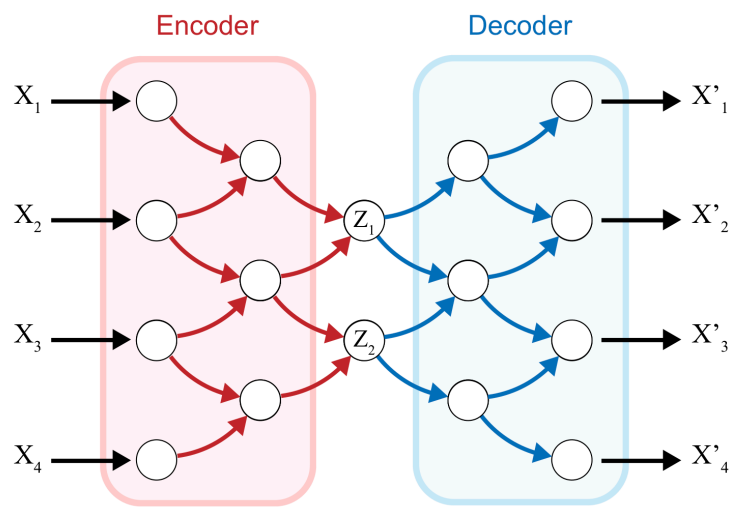

b) Predictive Coding

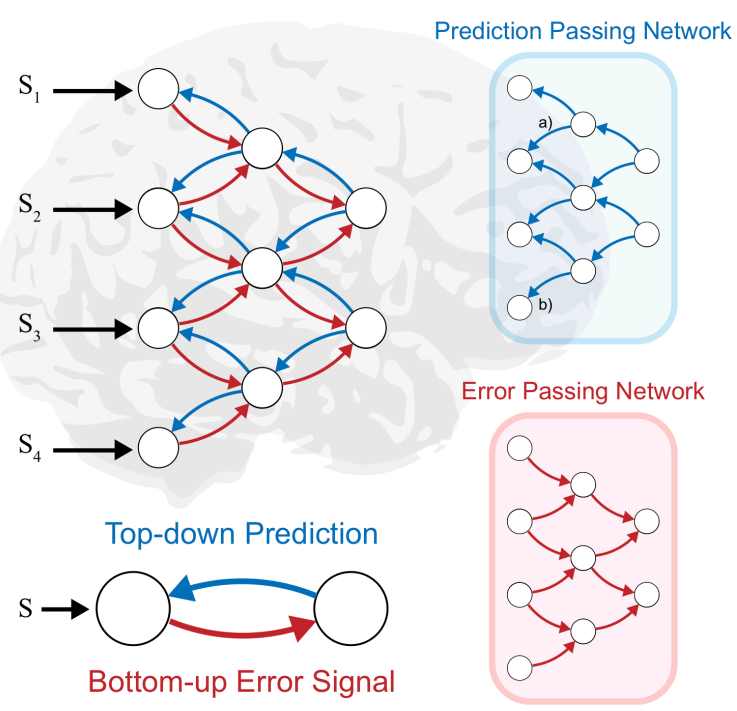

Figure 1. Comparison of information generation in $\mathbf{a})$ autoencoder and $\mathbf{b}$ ) predictive coding. a. In autoencoders, the encoder part (shown in red) compresses sensory information to compact representations in a latent space. This representation is decoded into sensory data format. The decoder (shown in blue) can be used for counterfactual information generation using a seed chosen from the latent space. The variables $z_{1}$ and $z_{2}$ represent the latent variables. $\mathbf{b}$. In the predictive coding hypothesis of the biological brain, bottom-up error signals (shown in red) correspond to data compression or encoding in autoencoders, whereas top-down predictions (shown in blue) correspond to information generation. Note that in predictive coding, our hypothesis predicts that the top-down predictions generate conscious experience. 